\title{
NOUVELLE
}

\section{Une mutation ponctuelle dans la protéine Rrp9 de la particule U3 entraîne un découplage des clivages précoces de I'ARN pré-ribosomique}

Mathieu Rederstorff

> Chez les eucaryotes, la biogenèse des ribosomes est un mécanisme complexe prenant place dans le nucléole [1] $(\rightarrow)$. L'assemblage et la maturation des sous-

$(\rightarrow)$ Voir la Synthèse de L. Tafforeau, $m / s$ $n^{\circ}$ 6-7, juin-juillet 2015, page 622

unités ribosomiques 405 et 605 y suivent deux routes indépendantes. Ces voies débutent néanmoins par la transcription, par l'ARN polymérase I, d'un ARN ribosomique (ARNr) précurseur commun, le pré-ARNr 35S. Des séries de clivages endo- et exo-nucléolytiques ordonnés permettent ensuite de libérer, à partir de ce précurseur, les futurs ARNr matures: I'ARNr 18S, de la petite sousunité ribosomique, d'une part, et les ARNr $5.8 \mathrm{~S}$ et $28 \mathrm{~S}$, d'autre part. Ces derniers, associés à l'ARNr $5 S$, transcrit de façon indépendante par l'ARN polymérase III, constitueront la grande sousunité ribosomique. Les clivages précoces du précurseur 355 ont lieu au sein d'un macro-complexe ribonucléoprotéique, le small subunit (SSU)-processome, ou pré-ribosome 90S, à l'origine de la petite sous-unité ribosomique 40S. Dans cette nouvelle, nous présentons les travaux récents portant sur le SSU-processome, réalisés notamment chez la levure Saccharomyces cerevisiae. Compte tenu de la conservation entre espèces et de l'universalité des processus de maturation des ARNr, ces résultats n'en revêtent pas moins un caractère crucial pour la compréhension de ce mécanisme dans l'espèce humaine.

Microscopie électronique : un bond immense dans l'étude du «processome ».

Les premières observations d'unités transcriptionnelles actives sur I'ADN ribosomique ont été possibles grâce aux expériences d' «étalements » de Miller (Miller spreads), dans les années 1960 [2] (Figure 1, en bas). Ces images de microscopie électronique « en sapin de Noël » révélaient la présence, sur les transcrits naissants, de boules termi-
Université de Lorraine, CNRS, IMoPA, F-54000 Nancy, France.

mathieu.rederstorff@univ-lorraine.fr

nales, dont la nature ne fut déterminée, en partie, que beaucoup plus tard: ces boules correspondent au processome en cours d'assemblage (Figure 1). Le processome final, ou pré-ribosome $90 \mathrm{~S}$, constitué, entre autres, de la petite particule ribonucléoprotéique nucléolaire (small nucleolar ribonucleoprotein particle, snoRNP) U3, comprend l'intégralité du pré-ARNr 35S.

Ces dernières années, des progrès considérables ont été réalisés dans la compréhension du mécanisme séquentiel d'assemblage du processome sur le pré-ARNr naissant [3, 4] (Figure 1), et dans la détermination des structures du complexe par cryo-microscopie électronique [4]. Plusieurs équipes ont notamment réussi à isoler, en utilisant des méthodes de purification par affinité avec des transcrits de longueur croissante, correspondant au long précurseur de l'ARNr 35S, les complexes s'associant de manière ordonnée et séquentielle à ce précurseur $[5,6]$. Une dernière étude a complété ces travaux, et montré qu'en 


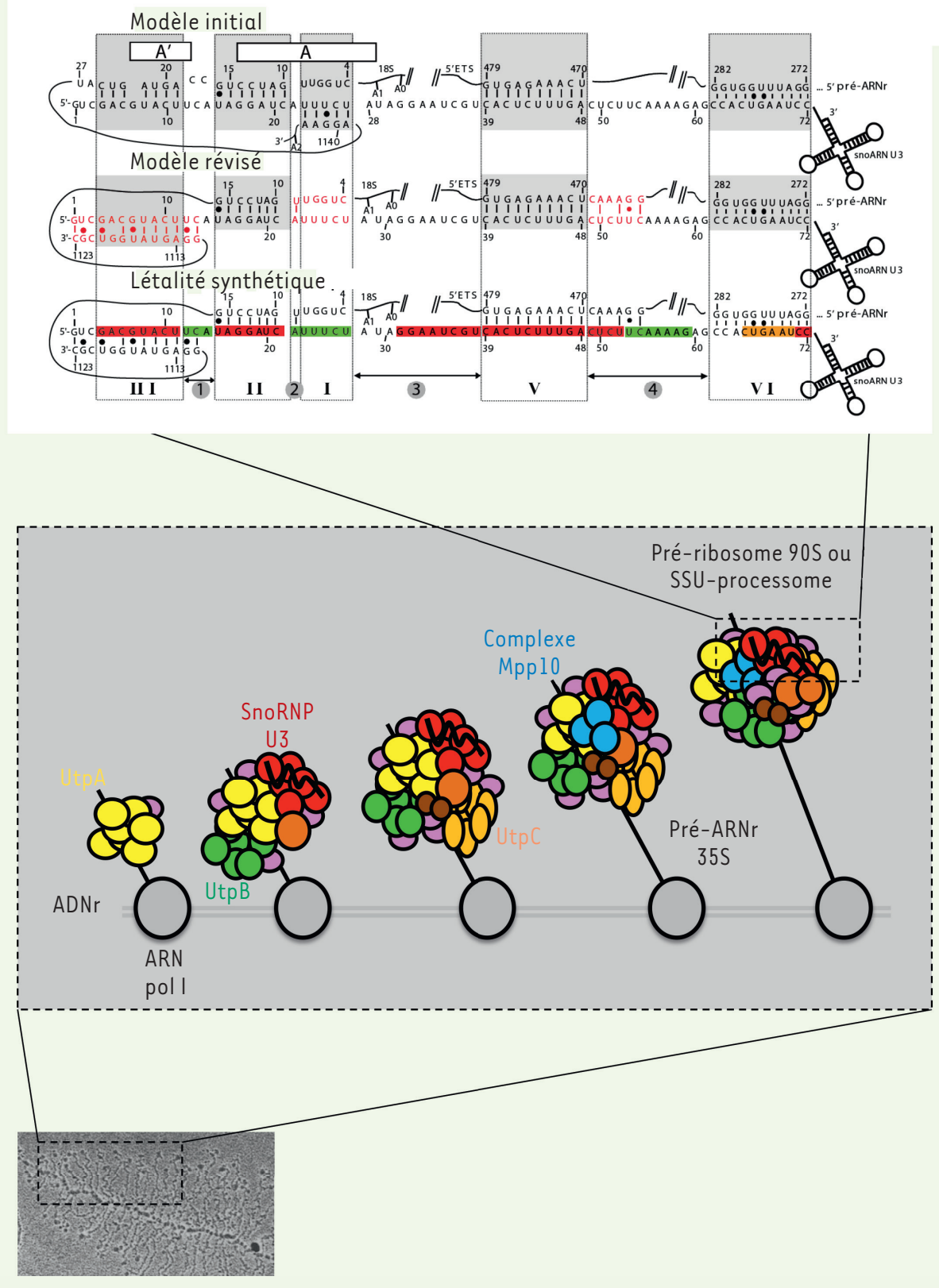

Figure 1. Assemblage séquentiel du « processome » et appariement entre le pré-ARN ribosomique et le petit ARN nucléolaire U3. (Bas) Visualisation d'un « étalement » de Miller (Miller spread) en microscopie électronique, correspondant à une unité transcriptionnelle d'ADN ribosomique en cours de transcription par l'ARN polymérase I (d'après [2]). Dans cette structure « en sapin de Noël », le tronc correspond à l'ADN ribosomique, les branches aux transcrits $35 \mathrm{~S}$ naissants, de longueur croissante de gauche à droite en fonction de l'avancement de la transcription, et les boules aux processomes en cours d'assemblage. (Milieu) Représentation schématique des «boutons terminaux » correspondant au processome en cours d'assemblage, sur les transcrits naissants de longueurs croissantes. La chronologie d'arrivée séquentielle des principaux sous-complexes du processome est illustrée [5, 6]. (Haut) Représentation de l'appariement entre le pré-ARN ribosomique et la région 5' fonctionnelle du petit ARN nucléolaire U3 (snoARN U3). Les hélices intermoléculaires III, II, I, V et VI, ainsi que les segments inter-hélices $1,2,3$ et 4 sont représentés. Les régions 5'-ETS (external transcribed spacer) et correspondant au futur $\mathrm{ARNr} 18 \mathrm{~S}$, ainsi que les sites de coupure précoce A0, $\mathrm{Al}$ et $\mathrm{A} 2$ sont indiqués, tout comme les positions de certains nucléotides. Les différences constatées entre le modèle initial et le modèle révisé sont indiquées en rouge dans ce dernier $[8,9]$. Enfin, le caractère synthétique létal (rouge), synthétique malade (orange), ou l'absence d'effet synthétique (vert) en présence du mutant de la protéine Rrp9 et des mutations dans le snoARN U3 correspondant aux régions en question sont illustrés dans le troisième appariement [10]. 
dépit de l'assemblage co-transcriptionnel des différents constituants du processome sur le pré-ARNr 35S, les régions du pré-ARNr transcrites en dernier (et non pas son extrémité 5' terminale) étaient intégrées les premières dans le processome [3]. Cela permet notamment d'éviter l'assemblage de particules comportant un transcrit abortif ou dégradé.

\section{La génétique de la levure en complément de la microscopie électronique}

Le petit ARN nucléolaire (small nucleolar RNA, snoRNA) U3, contrairement à la plupart des snoRNA à boîtes C/D [7] $(\rightarrow)$

$(\rightarrow)$ Voir la Synthèse de Y. Abel et al., $\mathrm{m} / \mathrm{s}$ $n^{\circ} 3$, mars 2014, page 297

n'est pas impliqué dans la méthylation d'autres ARN. Autre particularité : il est associé à une protéine qui lui est spécifique, la protéine Rrp9 chez la levure ou U3-55K chez l'homme. Enfin, le snoRNA U3 possède, à son extrémité 5', une extension qui n'existe pas chez les autres membres de la famille des snoRNA à boîtes $C / D$, et qui permet son appariement avec le pré-ARNr 35S (Figure 1, en haut). Au sein du processome, le snoRNP U3 est essentiel à la réalisation des clivages précoces $A 0, A l$ et $A 2$ permettant de libérer, à partir du précurseur 35S, I'ARNr 18 S mature de la petite sous-unité ribosomique. En effet, sur la base de comparaisons phylogéniques, de tests génétiques réalisés chez la levure, ou encore de l'utilisation de sondes chimiques in vivo, la nature de l'appariement entre le snoRNA U3 et le pré-ARNr 35 S a pu être établie (Figure I, en haut, ancien modèle). Cinq hélices intermoléculaires, séparées par quatre segments, ont ainsi pu être identifiées, et nommées à partir de l'extrémité 5' du snoRNA U3 : hélices III, II, et I d'une part, appariées au futur ARNr 18S, et hélices $V$ et VI d'autre part, appariées à la partie 5' ETS (external transcribed spacer) du pré-ARNr. L'importance fonctionnelle des hélices V, VI et II avait pu être montrée précédemment [8], notamment grâce à des expériences de mutations compensatoires, mais ces expériences n'avaient pas été concluantes pour les hélices I et III, et aucun test n'avait été réalisé sur les segments inter-hélices. Récemment, l'obtention des structures du processome par microscopie électronique a permis de confirmer l'existence des hélices VI, V et II, mais pas celle de I'hélice I, et de proposer l'existence d'une hélice III alternative (Figure 1, en haut, modèle révisé) [9]. Cela explique sans doute l'échec des précédentes tentatives d'étude des hélices I et III.

Néanmoins, une étude très récente a permis de faire la lumière sur certains aspects de la formation des hélices intermoléculaires entre le snoRNA U3 et le pré-ARNr et leur stabilisation. Cela a notamment été possible grâce à l'identification, chez la levure $S$. cerevisiae, d'un mutant inédit de la protéine Rrp9, qui entraîne un blocage et un découplage des coupures permettant de libérer I'ARNr 18S [10]. En outre, un criblage synthétique létal réalisé entre ce mutant et des mutants situés dans chaque hélice ou segment dans la région 5' fonctionnelle du snoRNA U3 a permis d'éclaircir le rôle de la protéine Rrp9 dans la formation et la stabilisation de l'appariement avec le pré-ARNr 35S, bien que le mécanisme mis en jeu soit probablement indirect (Figure 1, en haut, létalité synthétique) [10]. En effet, cette étude a permis de préciser le réseau d'interactions de la protéine Rrp9 au sein du processome, en identifiant de nouveaux partenaires de liaison de cette protéine, Rrp36 et Sgdl. L'absence de structure tridimensionnelle connue pour ces deux protéines ainsi que leur absence dans les structures de cryo-microscopie électronique actuelles ont conduit à proposer un nouveau modèle localisant Rrp36 et Sgdl au voisinage de Rrp9, bien défini dans ces structures [10].

Ainsi, la puissance des techniques actuelles de cryo-microscopie électronique et la précision des approches classiques de mutagenèse dirigée et de génétique chez la levure ont permis de franchir une nouvelle étape dans la compréhension du mécanisme d'assemblage de la petite sous-unité ribosomique. $\diamond$

Decoupling of pre-ribosomal RNA early cleavages by a point mutation in the U3 snoRNA-specific protein Rrp9

\section{LIENS D'INTÉRÊT}

L'auteur déclare n'avoir aucun lien d'intérêt concernant les données publiées dans cet article.

\section{RÉFÉRENCES}

1. Tafforeau L. Qu'en est-il de la biogenèse des ribosomes chez l'homme? Med Sci (Paris) 2015 ; 31 : 622-8.

2. Mougey $\varepsilon B, 0$ 'Reilly M, Osheim $Y$, et al. The terminal balls characteristic of eukaryotic rRNA transcription units in chromatin spreads are rRNA processing complexes. Genes Dev $1993 ; 7:$ 1609-19.

3. Cheng J, Baßler J, Fischer P, et al. Thermophile $90 \mathrm{~S}$ pre-ribosome structures reveal the reverse order of co-transcriptional 18S rRNA subdomain integration. Mol Cell 2019 ; 75 : 1256-69.e7.

4. Barandun J, Hunziker M, Klinge S. Assembly and structure of the SSU processome-a nucleolar precursor of the small ribosomal subunit. Curr Opin Struct Biol $2018 ; 49: 85-93$.

5. Chaker-Margot M, Hunziker M, Barandun J, et al. Stage-specific assembly events of the 6-MDa smallsubunit processome initiate eukaryotic ribosome biogenesis. Nat Struct Mol Biol 2015 ; 22 : 920-3.

6. Chen W, Xie Z, Yang F, Ye K. Stepwise assembly of the earliest precursors of large ribosomal subunits in yeast. Nucleic Acids Res 2017 ; 45 : 6837-47.

7. Abel Y, Clerget G, Bourguignon-Igel V, et al. Les petits ARN nucléolaires nous surprennent encore! Med Sci (Paris) 2014 ; $30: 297-302$.

8. Marmier-Gourrier N, Cléry A, Schlotter F, et al. A second base pair interaction between U3 small nucleolar RNA and the 5'-ETS region is required for early cleavage of the yeast pre-ribosomal RNA. Nucleic Acids Res 2011 ; 39 : 9731-45.

9. Sun $Q$, Zhu X, Qi J, et al. Molecular architecture of the 905 small subunit pre-ribosome. elife 2017 ; 6. doi : 10.7554/eLife.22086.

10. Clerget G, Bourguignon-Igel V, Marmier-Gourrier N, et al. Synergistic defects in pre-rRNA processing from mutations in the U3-specific protein Rrp9 and U3 snoRNA. Nucleic Acids Res $2020 ; 48$ : 3848-68.
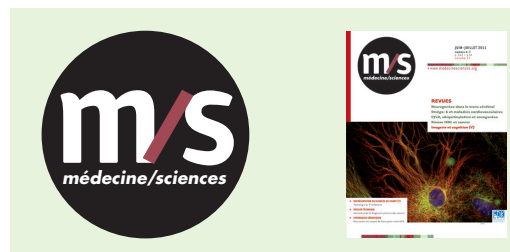

Abonnez-vous

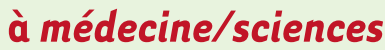

Bulletin d'abonnement page 426 dans ce numéro de $\mathrm{m} / \mathrm{s}$ 